\title{
A New Active Osseointegrated Implant System in Patients with Single-Sided Deafness
}

\author{
Kerstin Willenborg ${ }^{a}$ Emilio Avallone $^{a}$ Hannes Maier ${ }^{a, b}$ Thomas Lenarz ${ }^{a, b}$ \\ Susan Busch ${ }^{a}$ b

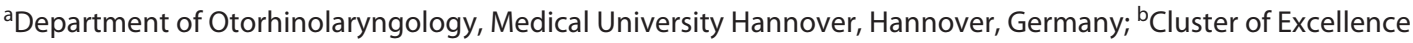 \\ Hearing4all, Medical University Hannover, Hannover, Germany
}

\section{Keywords}

Active bone conduction implant · Osia · Transcutaneous .

Single-sided deafness $\cdot$ Contralateral routing of signal

\begin{abstract}
Objective: The Cochlear ${ }^{\mathrm{TM}} \mathrm{Osia}^{\circledR}$ System (Osia) is an active transcutaneous bone conduction implant system intended for patients with conductive and mixed hearing loss but can also be used in cases of single-sided deafness (SSD) for the contralateral routing of signal (CROS). The Osia implant is placed subcutaneously under the intact skin behind the ear with the piezoelectric actuator connected to an osseointegrated BI300 implant - a titanium screw used for a 2-stage Baha surgery - on the mastoid. The external processor is magnetically attached to the subcutaneous implant receiver coil. As the Osia has recently been CE certified and is new on the market, with limited patient outcome data for SSD available, the objective of this study was the evaluation of surgical procedure, audiological results, and patient satisfaction for the Osia in SSD patients. Study Design: In a prospective, monocentric clinical observation study, 6 patients (18 years of age or older) with SSD and bone conduction thresholds
\end{abstract}

pure tone average $0.5,1,2$, and $4 \mathrm{kHz} \leq 25 \mathrm{~dB} \mathrm{HL}$ on the contralateral side were implanted with an Osia. Analysis of clinical outcome data with respect to surgical technique, adverse events, audiological measurement, and subjective benefit for SSD patients was conducted. Audiological measurements performed included hearing thresholds, sound field thresholds, word recognition scores (WRS; in \%) in quiet, and speech recognition thresholds in noise (in dB SNR). All tests were performed unaided and aided with the Osia. The subjective benefit with the Osia was determined by using 2 questionnaires; the Abbreviated Profile of Hearing Aid Benefit (APHAB) and the Bern Benefit in Single-Sided Deafness (BBSSD). Results: Preliminary results indicate a straightforward surgical procedure with a low rate of complications and an improvement in speech perception in quiet, listening performance in everyday situations and patient satisfaction. However, in one of 6 subjects, a revision surgery had to be performed. Conclusion: Provided that SSD patients are open for CROS hearing, they can benefit from the Osia by reduced head shadow effects and better speech recognition. Special caution should be given to the skin at the site of implantation to avoid complications.

(c) 2021 The Author(s)

Published by S. Karger AG, Basel karger@karger.com www.karger.com/aud

Karger $\stackrel{\text { ' }}{5}$

BOPEN ACCESS
(C) 2021 The Author(s)

Published by S. Karger AG, Basel

This is an Open Access article licensed under the Creative Commons Attribution-NonCommercial-4.0 International License (CC BY-NC) (http://www.karger.com/Services/OpenAccessLicense), applicable to the online version of the article only. Usage and distribution for commercial purposes requires written permission.
Correspondence to:

Susan Busch, busch.susan@mh-hannover.de 


\section{Introduction}

Patients with single-sided deafness (SSD) suffer from insufficient speech perception in noise and impaired localization of sound in part due to the head shadow effect [Welsh et al., 2004]. Binaural hearing can be partly restored with a cochlear implant (CI) [Kamal et al., 2012]. SSD patients implanted with CIs benefit from the bilateral input to the auditory system, which leads to improved speech in noise and reduced listening effort [Mertens et al., 2015]. But not all SSD patients are eligible for a cochlea implant, for example, children with agenesis of the auditory nerve and patients with a destructed inner ear or auditory nerve due to trauma, infection, or after surgery for vestibular schwannoma. Other treatment options for these patients are hearing systems for the contralateral routing of signals (CROS). A CROS system picks up the sound on the deaf side and transmits it to the normal hearing ear. CROS hearing can be provided with conventional hearing aids, a noninvasive treatment option, but patients have to wear 2 hearing aids. An alternative to CROS hearing with conventional hearing aids is percutaneous and transcutaneous bone conduction systems that transmit the sound directly to the skull bone, leading to transcranial CROS. In SSD patients, the results with bone conduction devices are similar [Snapp et al., 2017] or even superior [Ryu et al., 2015; Kim et al., 2017] compared to the outcome with conventional hearing aids. In percutaneous bone conduction systems like the Baha Connect (Cochlear, Sydney, Australia) and the Ponto (Oticon Medical, Askim, Sweden), a titanium implant is anchored in the mastoid bone and attached to a skin-penetrating abutment that is coupled to a vibration transducer transmitting the sound under the control of a sound processor [Hakansson et al., 1994]. The disadvantages of the percutaneous systems are skin reactions to the external abutment with high infection rates, especially in children; hypertrophic scarring with skin overgrowth; and fixture losses possibly leading to revision surgery or even explantation [House and Kutz, 2007; Gluth et al., 2010]. Although advances in the surgical procedure can notably reduce skin-related complications, they cannot be completely avoided [Calon et al., 2018; Kruyt et al., 2019].

In 2012, an active transcutaneous bone conduction system, the Bonebridge (MED-EL, Innsbruck, Austria), was introduced that was designed to overcome the problems of percutaneous systems. In this system, the bone conduction implant is placed under the skin and receives sound transmission by a processor that is held in place magnetically on the intact skin over the implant, thus

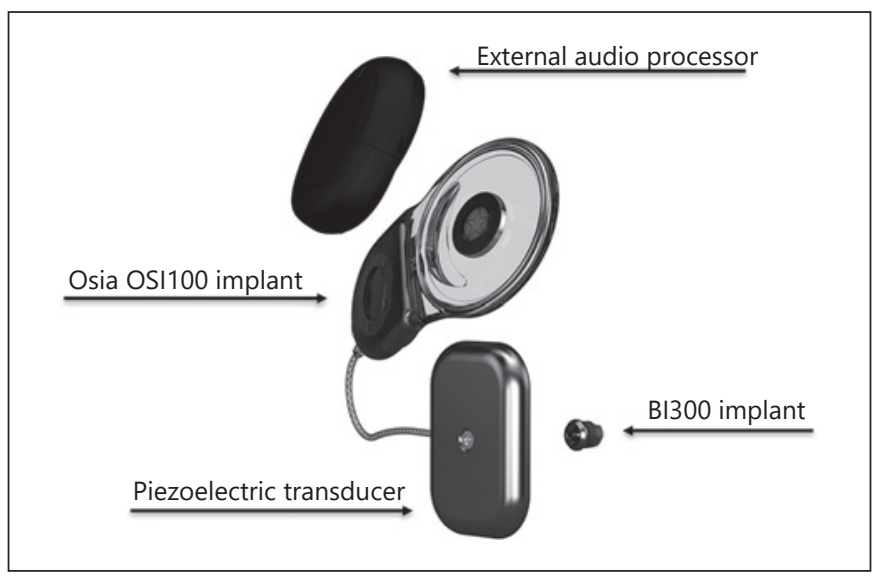

Fig. 1. Osia implant system and external audio processor (image by Cochlear Bone Anchored Solutions, Gothenburg, Sweden).

avoiding infections and the necessity to prevent skin overgrowth [Barbara et al., 2013; Sprinzl et al., 2013; Manrique et al., 2014]. The Bonebridge has been shown to have a low rate of adverse effects from surgery with a low percentage of explantation [Sprinzl and Wolf-Magele, 2016; Brkic et al., 2019] and has been successfully applied in the therapy of SSD [Salcher et al., 2017; Schmerber et al., 2017; Yang et al., 2018; Brkic et al., 2019].

The Cochlear ${ }^{\mathrm{TM}}$ Osia ${ }^{\circledR}$ System (Osia; Cochlear, Sydney, Australia) is a new active osseointegrated implant system intended for patients with conductive and mixed hearing loss but can be also used in cases of SSD. The Osia implant is placed subcutaneously under the intact skin behind the ear with the piezoelectric actuator connected to an osseointegrated BI300 implant on the mastoid. The external processor is magnetically attached to the subcutaneous implant receiver coil. The Osia is MRI-compatible at $1.5 \mathrm{~T}$ with the implant magnet and at $3 \mathrm{~T}$ after removal of the implant magnet. As the Osia has recently been CE certified and is new on the market, with limited patient outcome data for SSD available, the objective of this study was the evaluation of surgical procedure, audiological results, and patient satisfaction for the Osia in SSD patients.

\section{Methods}

Study Subjects

In this prospective, monocentric clinical observation study, subjects were qualified for enrollment if they were 18 years or older, suffered from SSD with a threshold of $\leq 25 \mathrm{~dB}$ HL (pure tone averages across $0.5,1,2$, and $4 \mathrm{kHz}\left[\mathrm{PTA}_{4}\right]$ ) on the contralateral
Willenborg/Avallone/Maier/Lenarz/ Busch 

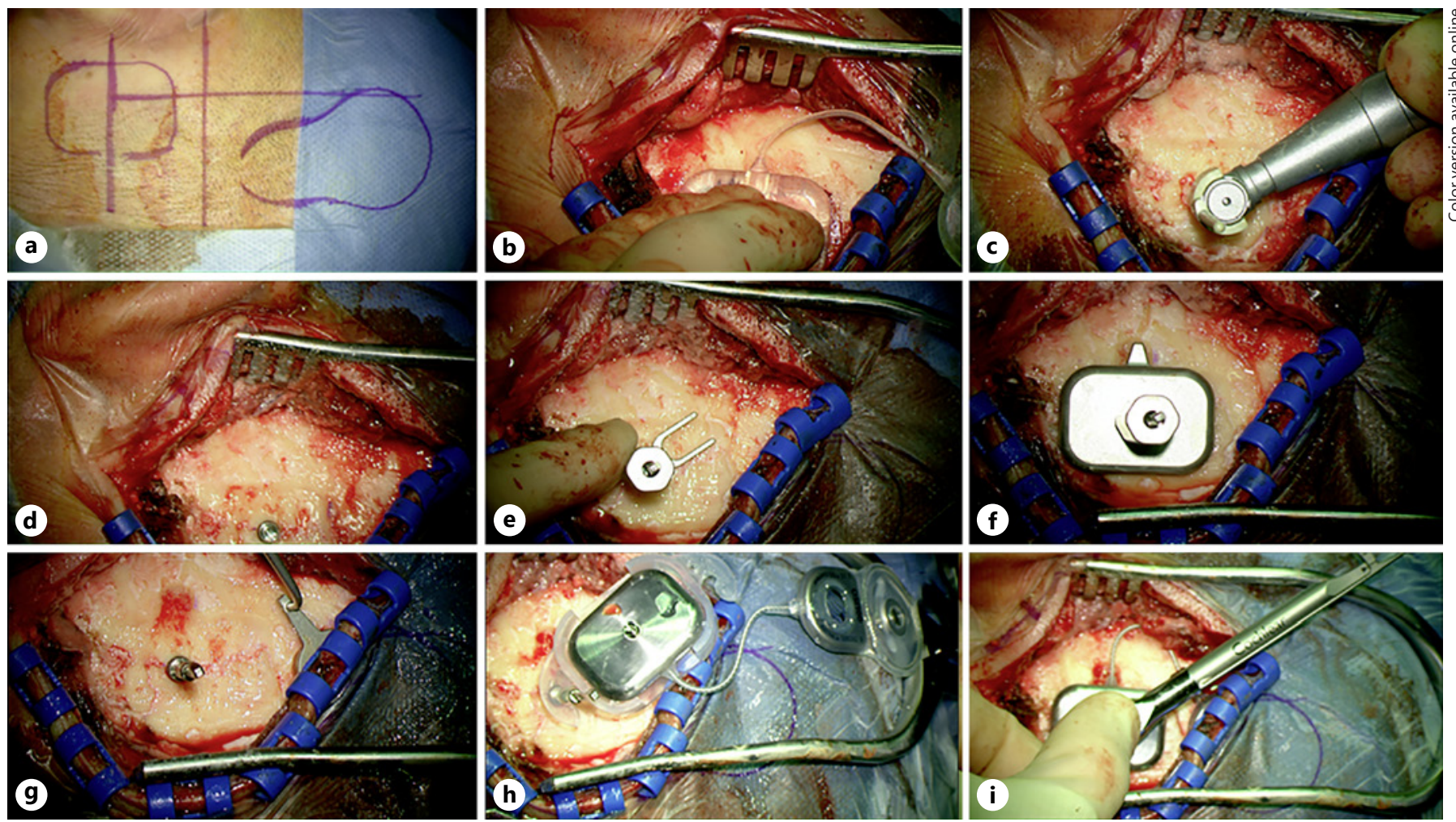

Fig. 2. Surgical steps of the Osia implantation in a left ear (auricle upward): planning of correct position of the implant components on the skin (a); marking of the position of the actuator on the bone (b); drilling with guide drill $(3 \mathrm{~mm} / 4 \mathrm{~mm})$ and widening drill (c); placing of the BI300 implant system (d); sliding the clearance indicator on the guide pin (e); marking of the position of the lead exit of the actuator (f); drilling of a recess for the actuator/receiver $(\mathbf{g})$; performing an integrity test $(\mathbf{h})$; fixing of the actuator (i).

side, and were not eligible for cochlear implantation. Patients gave their informed consent to participate in this study. This study has been approved by the local Ethics Committee (EC 8521_ MPG_23B_2019).

\section{Osia Implant}

The Osia comprises the bone conduction implant (OSI100) with a piezoelectric actuator, a BI300 implant, and an externally worn audio processor (Fig. 1). The audio processor receives the sound and transmits it to the OSI100, where the sound is converted by a piezoelectric element to mechanical vibrations applied to the skull bone with a BI300 titanium implant.

\section{Surgical Procedure}

Surgery was performed by KW under general anesthesia following the guidelines of the manufacturer. Prior to operation, computed tomography scans were performed for all patients to measure bone thickness and detect aberrant anatomical structures like an atypical sigmoid sinus in order to determine the optimal position for the implant and the possible insertion depth of the $\mathrm{BI} 300$. One of the 2 variants of the BI300 ( 3 and $4 \mathrm{~mm}$ ) that can be inserted deep into the bone, respectively, was chosen depending on available bone thickness. Computed tomography scans favored the placement of the BI300 in the sinodural angle, the position recommended by the manufacturer, in 5 patients. In one patient (subject 6), a retrosigmoidal position was chosen due to previous operations (CI and BAHA). After planning and marking the position of the components on the skin (Fig. 2a), a C-shaped flap containing skeletal muscle and the periosteum having the size of the actuator and a periosteal pocket for the receiver were created before marking of the BI300 implant site on the bone at the center of the actuator site (Fig. 2b). The removal of skeletal muscle and the periosteum over the actuator is necessary to prevent scar formation in the vicinity of the actuator that can impair its function. Then, a guide drill followed by a widening drill was used to drill to a depth of $3 \mathrm{~mm}$ or $4 \mathrm{~mm}$, depending on the thickness of the bone (Fig. 2c), followed by the insertion of the BI300 with the torque limited to 20-30 Ncm (Fig. 2d). A clearance indicator was then placed on a guide pin on the BI300 to assess the surface of the actuator site (Fig. 2e). Protruding bone was removed to avoid contact of the actuator with the bone in order to allow proper function. Similarly, an actuator template was used to mark the lead exit of the actuator to assess whether there is enough clearance (Fig. 2f). If necessary, a 2-mm deep V-shaped recess was drilled for the lead exit of the actuator. Then, a bone recess was created for the receiver (Fig. 2g), followed by an intraoperative integrity test (Fig. 2h) 
Table 1. Subject demographics and etiology

\begin{tabular}{|c|c|c|c|c|c|c|c|}
\hline Subject & Sex & $\begin{array}{l}\text { Age at implantation, } \\
\text { years }\end{array}$ & $\begin{array}{l}\text { Surditas/ } \\
\text { implanted side }\end{array}$ & Etiology & $\begin{array}{l}\mathrm{AC}_{\text {ipsi }} \mathrm{PTA}_{4} \\
\mathrm{~dB} \mathrm{HL}\end{array}$ & $\begin{array}{l}\text { ACcontra } \mathrm{PTA}_{4} \text {, } \\
\text { dB HL }\end{array}$ & $\begin{array}{l}\text { WRS }_{65} \\
\text { unaided, \% }\end{array}$ \\
\hline 1 & $\mathrm{~F}$ & 57.5 & $\mathrm{~L}$ & Congenital deafness & $>110$ & $8.8 \pm 7.5$ & 0 \\
\hline 2 & M & 44.4 & $\mathrm{~L}$ & Congenital deafness & $>109$ & $13.8 \pm 4.8$ & 0 \\
\hline 3 & M & 58.1 & $\mathrm{R}$ & Unknown; deafness since early childhood & $>110$ & $12.5 \pm 8.7$ & 0 \\
\hline 4 & $\mathrm{~F}$ & 50.1 & $\mathrm{R}$ & Deafness after otitis media at the age of 3 years & $>110$ & $11.3 \pm 6.3$ & 15 \\
\hline 5 & M & 53.5 & $\mathrm{R}$ & Unknown; deafness since early childhood & $>110$ & $13.8 \pm 4.8$ & 0 \\
\hline 6 & $\mathrm{~F}$ & 55.6 & $\mathrm{R}$ & Deafness after labyrinthitis/meningitis & $>109$ & $15.0 \pm 4.1$ & 0 \\
\hline
\end{tabular}

$\mathrm{AC}$, air conduction; $\mathrm{PTA}_{4}$, pure tone average $0.5,1,2,4 \mathrm{kHz}$; $\mathrm{WRS}_{65}$, word recognition score at $65 \mathrm{~dB}$ SPL; SPL, speech presentation level.

before fixing the actuator (Fig. 2i) and repositioning and suturing back the skeletal muscle, periosteum, and skin flap.

\section{Audiological Measurements}

Audiological measurements performed included pure tone audiometry, sound field audiograms $\left(\mathrm{S}_{0}\right)$, and the word recognition score (WRS; in \% at 65- $\mathrm{dB}$ speech presentation level [SPL] presentation level) in quiet using the Freiburg monosyllabic test. All tests were performed unaided and aided with the Osia in a sound-proof chamber with the speakers at a 1-m distance of the subject with the contralateral ear plugged and muffled. The program was set to the preferred everyday program with a microphone set to standard, zoom, or beam. Hearing thresholds of the contralateral ear were determined prior to the surgery and 1-4 months after the surgery. The speech performance was evaluated preoperatively unaided and postoperatively aided with the Osia 6 to 8 weeks after the initial fitting.

Additionally, speech intelligibility in noise (speech reception threshold, [SRT] in dB SNR) was determined with the German matrix sentence test (Oldenburg sentence test [OLSA]) with a fixed 65-dB SPL noise presented from the open normal hearing side and the speech (adaptive speech level) presented from the front ( $\left.\mathrm{S} 0 \mathrm{~N} 90_{\text {contra }}\right)$ and from the side of the implanted ear $\left(\mathrm{S} 90_{\mathrm{Os}-}\right.$ ia $N 90_{\text {contra }}$ ). The OLSA tests were performed unaided and aided 6 to 8 weeks after initial fitting.

The subjective benefit with the Osia in different listening situations/everyday situations was evaluated by using 2 questionnaires; the Abbreviated Profile of Hearing Aid Benefit (APHAB) [Cox and Alexander, 1995] and the Bern Benefit in Single-Sided Deafness (BBSSD) [Kompis et al., 2011]. Subjects filled in the questionnaire at the first control visit approximately 6-8 weeks after initial fitting and experience with the Osia.

\section{Statistics}

The small sample size of tested patients prevents exact statistical testing. Therefore, descriptive statistics were used to compare clinical outcome data, audiological measurements, and subjective benefit for SSD patients with the Osia in the unaided situation. If not otherwise indicated, means \pm standard deviation are shown. The $\mathrm{PTA}_{4}$ were calculated as mean values across the frequencies $0.5,1,2$, and $4 \mathrm{kHz}$.

\section{Results}

\section{Subjects}

In 2019, 6 subjects with SSD were implanted with the Osia (Table 1). The subjects were on average 53.2 ( \pm 5.2 ; standard deviation) years old and suffered from congenital hearing loss (2 cases), from hearing loss of unknown etiology since early childhood (3 cases) and from hearing loss due to labyrinthitis (1 case). One subject used a conventional hearing device (subject 3 ) and one other subject used a BAHA Connect (subject 6) for CROS hearing prior to the implantation with the Osia.

\section{Surgical Outcome}

Of the patients included in this study, 5 received an implant at the sinodural angle and one patient in a retrosigmoidal position (due to prior operations). The surgical procedure was fast, easily performed, and successful with no intraoperative complications. In one patient (subject 6), the implant had to be removed before initial fitting of the implant system due to wound dehiscence with pyogenic inflammation. Due to previous surgery at the site of implantation, the patient had a decreased thickness of subcutaneous tissue and a displaced temporal muscle.

\section{Safety}

The surgical procedure had no influence on the hearing threshold of the remaining ear as no changes in residual hearing were detected from preoperative thresholds (mean AC PTA $4=12.0 \pm 2.1 \mathrm{~dB} \mathrm{HL}$, Fig. 3) to postoperative thresholds (mean $\mathrm{AC} \mathrm{PTA} 4=12.0 \pm 2.0 \mathrm{~dB}$, Fig. 3).

\section{Audiological Results}

Of all 6 patients included in this study, the results of only 5 patients will be presented since one patient had to
Willenborg/Avallone/Maier/Lenarz/ Busch 


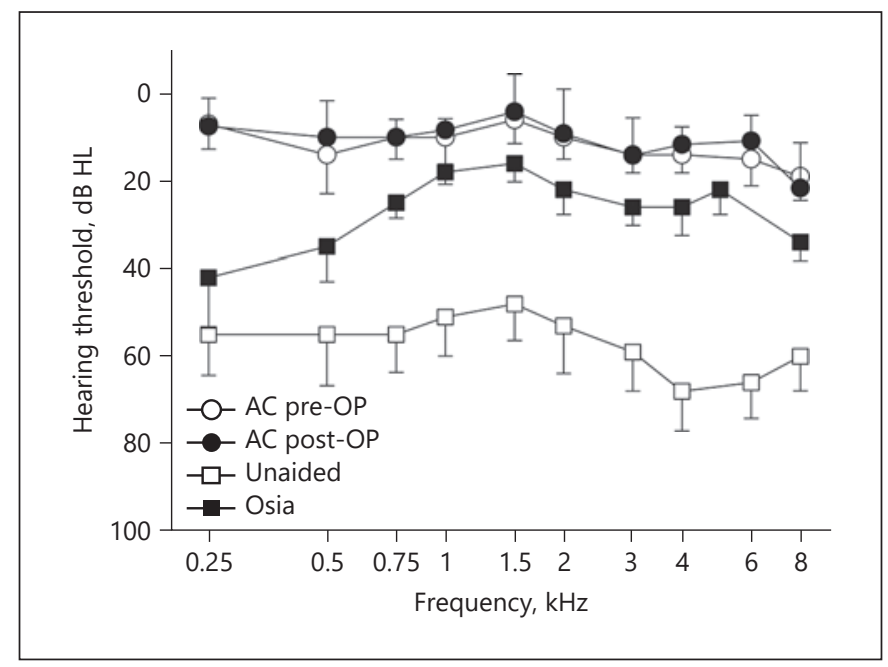

Fig. 3. Pure tone audiometry $(n=5)$. Mean pre- (AC pre-op, circles) and postoperative (AC post-op, black circles) air conduction thresholds of the (hearing) contralateral ear and thresholds of the deaf side unaided (unaided, white squares) and aided with the Osia (Osia, black squares) 3 months after surgery are shown \pm standard deviation.

be explanted before the implant activation (see Discussion). The mean pure tone threshold with the contralateral, normal hearing ear plugged and muffled $\left(\mathrm{PTA}_{4}\right) \mathrm{im}$ proved from $56.8 \pm 1.4 \mathrm{~dB}$ HL unaided to $25.3 \pm 2.2 \mathrm{~dB}$ HL aided with the Osia (Fig. 3).

Improvement in speech perception in quiet was tested with the Freiburg monosyllabic word test (Fig. 4). Mean WRSs (at $65 \mathrm{~dB}$, in \%) in quiet increased from $3.0 \pm 6.7 \%$ (unaided) to $95.0 \pm 3.5 \%$ (aided with Osia).

The OLSA test was used to monitor speech intelligibility in noisy environments with speech presented from different directions (Fig. 5). In the S0N90 ${ }_{\text {contra }}$ condition, where the speech is presented from the front and noise is applied to the contralateral (hearing) ear, the average SRT in noise improved from $-2.4 \pm 1.9 \mathrm{~dB}$ SNR unaided to $-4.5 \pm 1.3 \mathrm{~dB}$ SNR aided with the Osia, which is a benefit of $2.1 \mathrm{~dB}$ SNR for the subjects. In a more challenging $\mathrm{S} 90_{\text {Osia }} \mathrm{N} 90_{\text {contra }}$ condition, where the speech comes from the side of the deaf ear and the noise is applied to the normal hearing ear, the average SRT improved from $1.8 \pm 0.8$ $\mathrm{dB}$ SNR unaided to $-2.4 \pm 2.4 \mathrm{~dB}$ SNR aided with Osia. This is an improvement of $4.2 \mathrm{~dB}$ SNR for the subjects with the Osia.

\section{Subjective Benefit}

Subjective benefit with the Osia in everyday environment (ease of communication [EC], reverberation [RV],

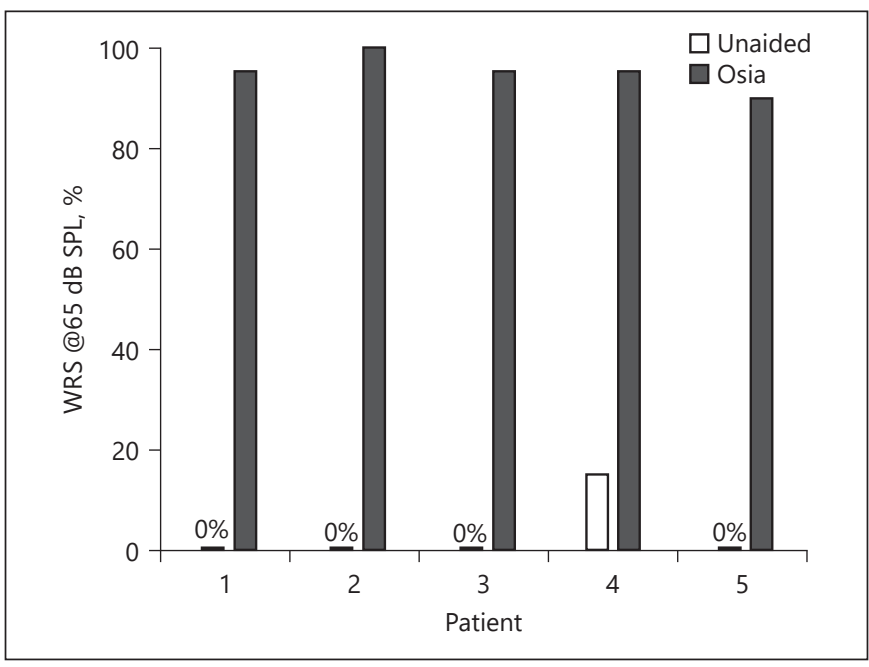

Fig. 4. WRSs at $65 \mathrm{~dB}$ SPL (in \%) of $n=5$ patients unaided (white bar) and aided with the Osia (black bar).WRS, word recognition score; SPL, speech presentation level.

and background noise $[\mathrm{BN}]$ ) and negative reactions to environmental noise (aversiveness [AV] of sounds) were rated using the $\mathrm{APHAB}$ and the BBSSD. Compared to the unaided situation, subjects experienced, on average, less problems with the Osia in the subscale EC ( $33 \%$ unaided vs. $15 \%$ Osia), $\mathrm{BN}$ ( $52 \%$ unaided vs. $28 \%$ Osia), and RV (57\% unaided vs. $35 \%$ Osia) (Fig. 6). The AV to loud sounds increased from unaided 19 to $34 \%$ aided with the Osia.

The mean score improvement of the BBSSD was 2.8, demonstrating a moderate benefit with the Osia. The scores range from 1.5 (question 9: directional hearing) to 4.2 (question 6: talk in a moving car). The mean overall hearing (question 10) determined for all subjects was 3.5 $( \pm 1.2)$ (Fig. 7).

Subjective benefit and satisfaction are also reflected in the wearing time of the device. After 6 to 8 weeks, 4 subjects used the implant system between 8 and $16 \mathrm{~h}$ per day, and 1 subject (Subject 4 ) used the device for 2 to $3 \mathrm{~h}$ because of magnet strength-related discomfort with the head-worn processor. After reducing magnet strength, the subject felt instantly better and the wearing time increased up to $4 \mathrm{~h}$ per day. A slight discomfort after a 4 -h wearing time could be a sign that the magnet strengths need to be further reduced to prolong wearing time. 
Fig. 5. Displayed are the results of the OLSA in noise $(n=5) . \mathrm{SRT}_{50}$ in $65-\mathrm{dB}$ noise for $n=5$ subjects unaided (white bars) and aided with the Osia (black bars) for the measurement setup $\mathrm{S} 0 \mathrm{~N} 90_{\text {contra }}(\mathbf{a})$ and S90 Osia $\mathrm{N} 90_{\text {contra }}$ (b). OLSA, Oldenburg sentence test; SRT, speech reception threshold.
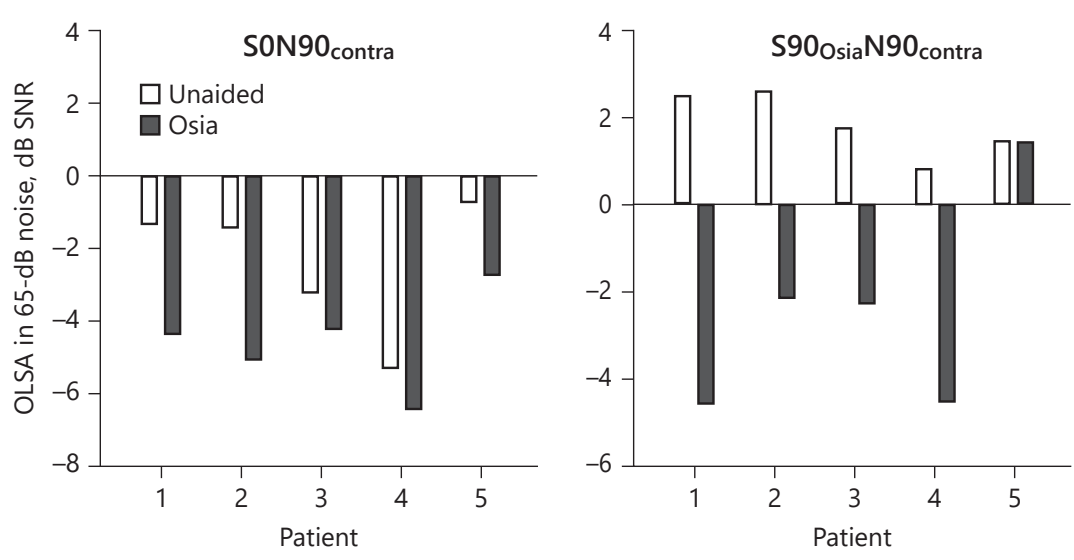

a

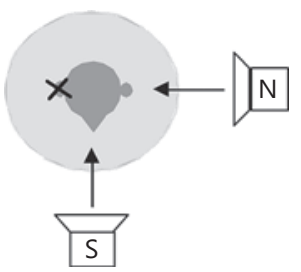

\& Unaided

+ Osia

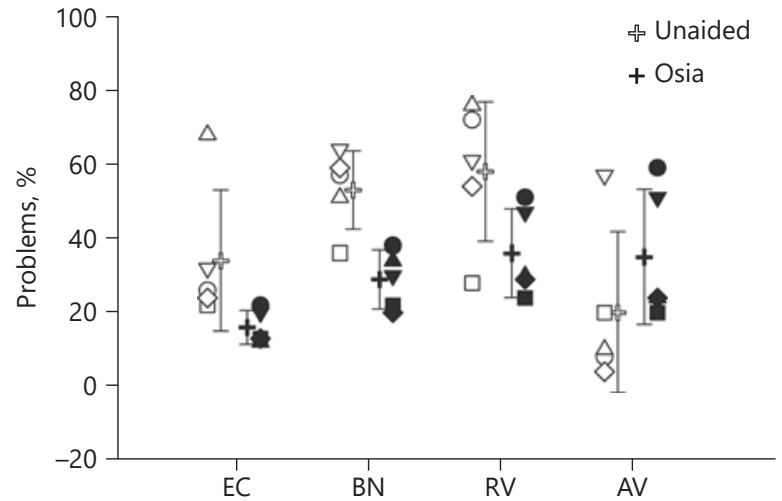

Fig. 6. Subjective benefit measured with the questionnaire. Mean values ( \pm standard deviation) of 5 subjects and single subject scores for the categories $\mathrm{EC}, \mathrm{BN}, \mathrm{RV}$, and $\mathrm{AV}$ to loud sounds preoperatively (white symbols) and postoperatively (black symbols). EC, ease of communication; $\mathrm{BN}$, background noise; RV, reverberation; AV, aversiveness; APHAB, Abbreviated Profile of Hearing Aid Benefit.

\section{Discussion}

This study demonstrates that the Osia can be implanted safely in patients with SSD with a subjective and objective benefit. To this end, 6 patients with SSD received the new transcutaneous bone conduction implant Osia. The surgical procedure was successful in all patients with no intraoperative complications, although one patient (subject 6) had to be explanted prior to the initial fitting of the implant due to wound dehiscence with pyogenic inflammation. This patient had previously been implanted with a CI that had to be revised and later explanted. Simultaneously, with the CI explantation, the patient had been implanted with a Baha Connect that had to be removed due to wound infection and skin overgrowth. Because of the multiple previous operations close to the implantation site with thinned subcutaneous tissue and displaced temporal muscle, the wound healing deficit was probably due to decreased thickness and impaired blood flow and not related to the surgical procedure or the implant. Thus, although only few patients were included in this study, one might expect the frequency of complications to be similarly low as for the other commercially available transcutaneous bone conduction implant, the Bonebridge, because the surgical procedure is similar. How- 
Fig. 7. Subjective benefit measured with the BBSSD. Mean results ( \pm standard deviation) of 5 subjects with the Osia compared to the unaided situation are shown. Ratings may vary between similar with or without Osia ( 0$)$, easier with Osia $(>0$ to +5$)$, and easier without Osia $(<0$ to -5$)$. BBSSD, Bern Benefit in Single-Sided Deafness Questionnaire.

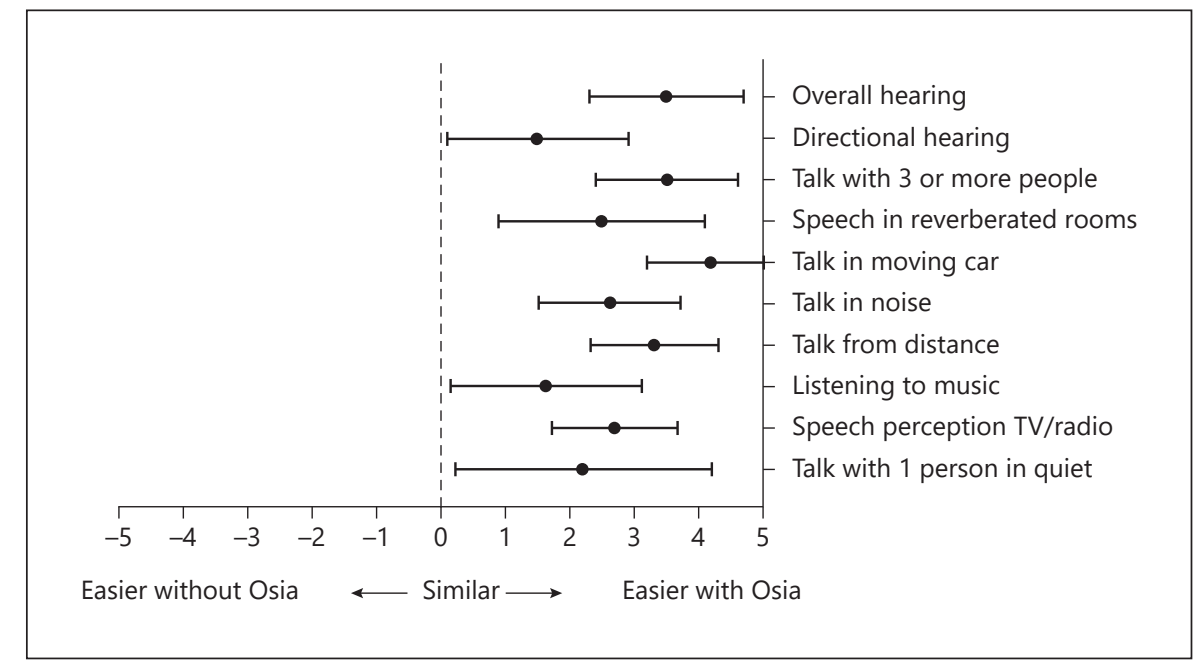

ever, the increased size, especially the increased protrusion above the level of the bone in the Osia when compared to the Bonebridge, may influence the healing process and compatibility of the implant. Further studies will have to show whether there are significant differences in the complication rates between the 2 transcutaneous devices or in comparison with other bone-anchored hearing systems. Nevertheless, one might consider avoiding an implantation in a skin region compromised by previous surgeries with impaired blood supply and reduced thickness, and the decision about the placement of the implant should take into consideration previous surgical procedures. We would recommend placing the implant out of the region of previous surgery, especially behind the mastoid.

Patients with single-sided deafness suffer from insufficient speech perception in noise due to the head shadow effect [Welsh et al., 2004]. When applying a CROS device as a solution in SSD, it needs to compensate for the predominant high-frequency deficit caused by the head shadow effect [Shaw, 1974]. With a bone conduction device as a CROS solution, the setting is further influenced by frequency-specific transcranial attenuation [Nolan and Lyon, 1981], which is more pronounced at higher frequencies than at lower frequencies.

Preliminary results demonstrate substantial improvements in pure tone audiometry and speech perception in quiet and listening performance in everyday situations with the Osia compared to the unaided situation. The mean pure tone threshold improved by $31.5 \mathrm{~dB}$ for subjects aided with the Osia, and the contralateral (hearing) ear plugged and muffled signifies that sufficient high-frequency information is provided by the Osia. The mean pure tone threshold with the Osia is in the same range as in a study by Salcher et al. [2017] of 10 patients with the Bonebridge in SSD who showed an improvement in mean pure tone threshold of $36 \mathrm{~dB}$. Also, Brkic et al. [2019] described a similar outcome in the pure tone audiometric thresholds in 20 patients with SSD after implantation of a Bonebridge.

To test speech recognition, the Freiburg monosyllabic test in quiet and the OLSA in quiet and noise were applied. The Freiburg monosyllabic test has been the gold standard for speech recognition in German language for many years. The OLSA allows the simulation of real-life relevant hearing situations. In the Freiburg monosyllabic test, mean WRSs (in \% at $65 \mathrm{~dB}$ SPL) increased significantly from $2.5 \pm 6.1 \%$ (unaided) to $93.0 \pm 7.6 \%$ (aided with Osia). This is in line with previous results. Salcher et al. [2017] described an increase in the WRS from $8 \%$ before implantation to $84 \%$ after implantation of the Bonebridge, while Brkic et al. [2019] reported a mean hearing gain of $49 \%$ in the WRS in the same setting.

Furthermore, the OLSA was used to monitor relevant hearing situations. In the setting with sound coming from the front and noise applied to the contralateral (hearing) ear, our results showed a significant improvement of -2.1 $\mathrm{dB}$ SNR. In the more challenging setting with sound coming from the side of the deaf ear and noise applied to the contralateral (hearing) ear, our results showed an even stronger improvement of $-4.2 \mathrm{~dB}$ SNR. Only subject 5 showed similar results for the unaided and aided OLSA. The aided OLSA (S90N90) has been the last test performed in the late afternoon, and fatigue and a lack of focus and concentration might have led to a decrement in performance and comparable results. 
These results are in accordance with previous studies on active transcutaneous and percutaneous implants. Salcher et al. [2017] found a mean increase in the SNR of $-2.5 \mathrm{~dB}$ under the same challenging conditions, while Laske et al. [2015] described a significant improvement of $-1.7 \mathrm{~dB}$ SNR with speech coming from the deaf side and noise applied from the front. Although we could demonstrate a clear benefit with the OLSA in the aforementioned configurations, we are also aware of situations, for example, the noise presented to the deaf side and transmitted to the better hearing ear, in which a CROS system is less beneficial and the improvement in speech intelligibility in noise is limited [Hol et al., 2010] or even impaired [Lin et al., 2006; Finbow et al., 2015; Ryu et al., 2015].

Subjective benefit was assessed using 2 established questionnaires. The results from the APHAB showed a subjective improvement in $\mathrm{EC}, \mathrm{BN}$, and $\mathrm{RV}$ items in all patients, while AV to loud sounds increased in only 3 patients, was unchanged in one patient, and decreased in one patient. Salcher et al. [2017] reported similar results with Bonebridge in SSD concerning EC, BN, and RV, but no difference in AV to loud sounds was found. The increase in AV to loud sounds with the Osia in our study is not unusual. It was also observed after hearing rehabilitation of SSD patients with other bone conduction implants [Linstrom et al., 2009; House et al., 2010; Faber et al., 2013; Gawecki et al., 2016]. AV to loud sounds may decline over time as patients acclimate to the new hearing situation. According to the BBSSD, all patients experienced subjective improvement after Osia implantation. BBSSD scores showed an advantage of the Osia in the most challenging environments for speech understanding such as conversations in a moving car, conversation in a group ( $\geq 3$ people), or conversations at greater distances. These results are comparable to Laske et al. [2015] and Salcher et al. [2017] where most patients implanted with a Bonebridge in SSD reported a subjective benefit in a similar setting.

\section{Conclusion}

Our preliminary results show that the Osia is a new active transcutaneous bone conduction implant that can be easily implanted, though skin-related issues led to 1 revision surgery after implantation. Subjective and objective functional benefits in patients with SSD are comparable to the Bonebridge, the other commercially available active bone conduction implant. Thus, SSD patients who are not eligible for a CI can benefit from the Osia. How- ever, implantation should not be performed in a skin region compromised by previous surgeries with impaired blood supply and reduced thickness.

\section{Statement of Ethics}

This study has been approved by the local Ethics Committee of the Hannover Medical School (EC 8521_MPG_23B_2019). The patients gave their informed consent to participate in this study.

\section{Conflict of Interest Statement}

The authors declare that the research was conducted in the absence of any commercial or financial relationships that could be construed as a potential conflict of interest.

\section{Funding Sources}

This work was supported by the DFG Cluster of Excellence EXC 2177 "Hearing4all 2.0" Project ID 390895286. K.W., S.B., and H.M. received the travel support to meetings from Cochlear. No funds were received for this work from the National Institutes of Health (NIH), Wellcome Trust, or Howard Hughes Medical Institute (HHMI).

\section{Author Contributions}

All authors contributed equally to this work: T.L. and K.W. conducted the surgery; S.B. and H.M. designed the study; S.B. conducted the study, and collected and analyzed the data. K.W. and S.B. wrote the main manuscript; and all authors discussed the results and implications, and commented on the manuscript at all stages.

References

Barbara M, Perotti M, Gioia B, Volpini L, Monini S. Transcutaneous bone-conduction hearing device: audiological and surgical aspects in a first series of patients with mixed hearing loss. Acta Otolaryngol. 2013 Oct;133(10):1058-64.

Brkic FF, Riss D, Scheuba K, Arnoldner C, Gstöttner W, Baumgartner WD, et al. Medical, technical and audiological outcomes of hearing rehabilitation with the Bonebridge transcutaneous bone-conduction implant: a single-center experience. J Clin Med. 2019 Oct 3;8(10): 1614 .

Calon TGA, Johansson ML, de Bruijn AJG, van den Berge H, Wagenaar M, Eichhorn E, et al. Minimally invasive ponto surgery versus the linear incision technique with soft tissue preservation for bone conduction hearing implants: a multicenter randomized controlled trial. Otol Neurotol. 2018 Aug;39(7):882-93.
90

Audiol Neurotol 2022;27:83-91

DOI: $10.1159 / 000515489$
Willenborg/Avallone/Maier/Lenarz/ Busch 
Cox RM, Alexander GC. The abbreviated profile of hearing aid benefit. Ear Hear. 1995 Apr; 16(2):176-86

Faber HT, de Wolf MJ, Cremers CW, Snik AF, Hol MK. Benefit of Baha in the elderly with single-sided deafness. Eur Arch Otorhinolaryngol. 2013 Mar;270(4):1285-91.

Finbow J, Bance M, Aiken S, Gulliver M, Verge J, Caissie R. A comparison between wireless CROS and bone-anchored hearing devices for single-sided deafness: a pilot study. Otol Neurotol. 2015 Jun;36(5):819-25.

Gawecki W, Stieler OM, Balcerowiak A, Komar D, Gibasiewicz R, Karlik M, et al. Surgical, functional and audiological evaluation of new $\mathrm{Baha}((\mathrm{R}))$ Attract system implantations. Eur Arch Otorhinolaryngol. 2016 Oct;273(10): 3123-30.

Gluth MB, Eager KM, Eikelboom RH, Atlas MD. Long-term benefit perception, complications, and device malfunction rate of bone-anchored hearing aid implantation for profound unilateral sensorineural hearing loss. Otol Neurotol. 2010 Dec;31(9): 1427-34.

Hakansson BE, Carlsson PU, Tjellstrom A, Liden G. The bone-anchored hearing aid: principal design and audiometric results. Ear Nose Throat J. 1994 Sep;73(9):670-5.

Hol MK, Kunst SJ, Snik AF, Cremers CW. Pilot study on the effectiveness of the conventional CROS, the transcranial CROS and the BAHA transcranial CROS in adults with unilateral inner ear deafness. Eur Arch Otorhinolaryngol. 2010 Jun;267(6):889-96.

House JW, Kutz JW Jr. Bone-anchored hearing aids: incidence and management of postoperative complications. Otol Neurotol. 2007 Feb;28(2):213-7.

House JW, Kutz JW Jr, Chung J, Fisher LM. Boneanchored hearing aid subjective benefit for unilateral deafness. Laryngoscope. $2010 \mathrm{Mar}$ 120(3):601-7.

Kamal SM, Robinson AD, Diaz RC. Cochlear implantation in single-sided deafness for enhancement of sound localization and speech perception. Curr Opin Otolaryngol Head Neck Surg. 2012 Oct;20(5):393-7.
Kim G, Ju HM, Lee SH, Kim HS, Kwon JA, Seo YJ. Efficacy of bone-anchored hearing aids in single-sided deafness: a systematic review. Otol Neurotol. 2017 Apr;38(4):473-83.

Kompis M, Pfiffner F, Krebs M, Caversaccio MD Factors influencing the decision for Baha in unilateral deafness: the Bern benefit in singlesided deafness questionnaire. Adv Otorhinolaryngol. 2011;71:103-11.

Kruyt IJ, Kok H, Bosman A, Nelissen RC, Mylanus EAM, Hol MKS. Three-year clinical and audiological outcomes of percutaneous implants for bone conduction devices: comparison between tissue preservation technique and tissue reduction technique. Otol Neurotol. 2019 Mar;40(3):335-43.

Laske RD, Röösli C, Pfiffner F, Veraguth D, Huber AM. Functional results and subjective benefit of a transcutaneous bone conduction device in patients with single-sided deafness. Otol Neurotol. 2015 Aug;36(7):1151-6.

Lin LM, Bowditch S, Anderson MJ, May B, Cox KM, Niparko JK. Amplification in the rehabilitation of unilateral deafness: speech in noise and directional hearing effects with bone-anchored hearing and contralateral routing of signal amplification. Otol Neurotol. $2006 \mathrm{Feb}$;27(2):172-82.

Linstrom CJ, Silverman CA, Yu GP. Efficacy of the bone-anchored hearing aid for single-sided deafness. Laryngoscope. 2009 Apr;119(4): $713-20$.

Manrique M, Sanhueza I, Manrique R, de Abajo J. A new bone conduction implant: surgical technique and results. Otol Neurotol. 2014 Feb;35(2):216-20.

Mertens G, Punte AK, De Bodt M, Van de Heyning P. Binaural auditory outcomes in patients with postlingual profound unilateral hearing loss: 3 years after cochlear implantation. Audiol Neurotol. 2015;20(1):67-72.

Nolan M, Lyon DJ. Transcranial attenuation in bone conduction audiometry. J Laryngol Otol. 1981 Jun;95(6):597-608.
Ryu NG, Moon IJ, Byun H, Jin SH, Park H, Jang KS, et al. Clinical effectiveness of wireless CROS (contralateral routing of offside signals) hearing aids. Eur Arch Otorhinolaryngol. 2015 Sep;272(9):2213-9.

Salcher R, Zimmermann D, Giere T, Lenarz T, Maier H. Audiological results in SSD with an active transcutaneous bone conduction implant at a retrosigmoidal position. Otol Neurotol. 2017 Jun;38(5):642-7.

Schmerber S, Deguine O, Marx M, Van de Heyning P, Sterkers O, Mosnier I, et al. Safety and effectiveness of the Bonebridge transcutaneous active direct-drive bone-conduction hearing implant at 1-year device use. Eur Arch Otorhinolaryngol. 2017 Apr;274(4): 1835-51.

Shaw EAG. Transformation of sound pressure level from the free field to the eardrum in the horizontal plane. J Acoust Soc Am. 1974 Dec; 56(6): 1848-61.

Snapp HA, Holt FD, Liu X, Rajguru SM. Comparison of speech-in-noise and localization benefits in unilateral hearing loss subjects using contralateral routing of signal hearing aids or bone-anchored implants. Otol Neurotol. 2017 Jan;38(1):11-8

Sprinzl G, Lenarz T, Ernst A, Hagen R, WolfMagele A, Mojallal H, et al. First European multicenter results with a new transcutaneous bone conduction hearing implant system: short-term safety and efficacy. Otol Neurotol. 2013 Aug;34(6):1076-83.

Sprinzl GM, Wolf-Magele A. The Bonebridge bone conduction hearing implant: indication criteria, surgery and a systematic review of the literature. Clin Otolaryngol. 2016 Apr;41(2): 131-43.

Welsh LW, Welsh JJ, Rosen LF, Dragonette JE. Functional impairments due to unilateral deafness. Ann Otol Rhinol Laryngol. 2004 Dec;113(12):987-93.

Yang J, Wang Z, Huang M, Chai Y, Jia H, Wu Y, et al. BoneBridge implantation in patients with single-sided deafness resulting from vestibular schwannoma resection: objective and subjective benefit evaluations. Acta Otolaryngol. 2018 Oct;138(10):877-85. 